\title{
On the canonical algebra of smoothings of sandwiched singularities
}

\author{
T. de Jong
}

\begin{abstract}
In this paper we relate the possible finite generatedness of the canonical algebra of smoothings of sandwiched singularities (Kollár's conjecture) to the possible finite generatedness of the symbolic algebra of certain curves in three-space.
\end{abstract}

\section{Introduction}

This paper is on a discussion of a conjecture of Kollár for the special case of sandwiched singularities. We recall this conjecture.

Conjecture 1 (Kollár [Kol91, $\S 6.2 .1]$ ). Let $\mathbf{X}$ be a smoothing of a rational surface singularity $X$, and $K_{\mathbf{X}}$ be the canonical divisor of $\mathbf{X}$. Then the canonical algebra

$$
R:=\bigoplus_{k=0}^{\infty} \mathcal{O}_{\mathbf{X}}\left(k \cdot K_{\mathbf{X}}\right)
$$

is finitely generated.

The finite generatedness of the canonical algebra is equivalent to the existence of some kind of modification. In fact we have the following proposition.

Proposition 2. Let $\mathbf{X}$ be a smoothing of a rational surface singularity $X$, and $K_{\mathbf{X}}$ be the canonical divisor of $\mathbf{X}$. Then the following are equivalent.

1) The canonical algebra is finitely generated.

2) There exists a modification $\pi: \mathbf{W}:=\operatorname{Proj}(R) \longrightarrow \mathbf{X}$ with the following properties:

a) $\pi$ is small, that is, $\pi^{-1}(x)$ is one dimensional for $x$ the singular point of $\mathbf{X}$;

b) $K_{\mathbf{W}}$ is $\pi$-ample;

c) some multiple $m K_{\mathbf{W}}$ is Cartier, that is, $K_{\mathbf{W}}$ is $\mathbb{Q}$-Cartier.

For a proof, we refer to [Kaw88]. Restricting $\pi$ to the special fiber would give a modification $\pi$ : $W \longrightarrow X$, with $K_{W} \pi$-ample, and such that all singularities of $W$ are $q G$-singularities: singularities for which the canonical cover has an equivariant smoothing. Kollár's conjecture would imply that one can get all smoothings of $X$ by considering smoothings of partial resolutions. Several special cases of Kollár's conjecture are known.

1) The case of smoothings over the Artin component (see [Art70]). In this case the smoothings are induced by deformations of the rational double point resolution. In particular the conjecture holds for rational double and triple points. 


\section{CANONICAL ALGEBRA OF SMOOTHINGS OF SANDWICHED SINGULARITIES}

2) Smoothings of quotient surface singularities, and of quotients of simple elliptic and cusp singularities (see [KS88], ch. 3 and 5). These results of Kollár and Shepherd-Barron are obtained by using deep results in three-dimensional geometry (Mori's program).

3) Smoothings of rational quadruple points (see Stevens [Ste91]). This case is proved by constructing partial resolutions for each smoothing component. The number of smoothing components for rational quadruple points are known by the results of [JS91].

Although the conjecture is more than ten years old now, there has not been any progress since then. The problem seems to be extremely difficult. In this paper we discuss Kollár's conjecture for sandwiched singularities, a class of singularities introduced by Hironaka [Hir83]. We relate the finite generatedness of the canonical algebra with the finite generatedness of the symbolic algebra of a curve in three-space: this makes the problem much more geometric and it is expected that the problem is now easier to handle, certainly in practice.

Sandwiched singularities, by definition, are singularities which occur on a blowing-up of $\mathbb{C}^{2}$ in a complete ideal. Sandwiched singularities were studied by, for example, Lipman [Lip69] and Spivakovsky [Spi90]. These sandwiched singularities build a big class of rational surface singularities, and can be seen as generalizations of the $A_{k}$-singularities.

In [JS98] the deformation theory of sandwiched singularities is studied. To describe the result we need, we note that sandwiched singularities can also be obtained by considering decorated curves $(C, \ell)$ : an isolated plane curve singularity $C=\bigcup_{i \in T} C_{i}$, together with a function $\ell$ which assigns to each branch a natural number, satisfying the condition $\ell(i) \geqslant m(i)$. Here $m(i)$ is the sum of the multiplicities of branch $C_{i}$ in the minimal (not necessarily good) embedded resolution of $C$.

To $\ell$ we can associate for each $i \in T$ a subscheme of length $\ell(i)+c(i)$ on the normalization of $C_{i}$ at the point which maps to the singular point of $C$. Here $c(i)$ is the $i$ th conductor number. This subscheme can be cut out by a function $g \in \mathbb{C}\{x, y\}$. We obtain the sandwiched space $X(C, \ell)$ by taking the minimal (not necessarily good) embedded resolution of $C$ and then doing $\ell(i)-m(i)$ extra successive blowing-ups at the intersection points of the strict transform of $C_{i}$. Then blow down all exceptional curves not intersecting the strict transform of $C_{i}$ for each $i$. Denote $M(i)$ as the sum of the multiplicities of branch $C_{i}$ in the minimal embedded good resolution of $C$. If moreover $\ell(i) \geqslant M(i)+1$, the sandwiched space has a unique singularity. This is a sufficient, but in general not a necessary condition. Still another way to get $X(C, \ell)$ is by normalizing the surface singularity $Y(C, \ell)$ in $\left(\mathbb{C}^{3}, 0\right)$, given by the equation $z f-g=0$.

General one-parameter (parameter $t$ ) smoothings of $X(C, \ell)$ can be seen geometrically by oneparameter $\delta$-constant deformations $\mathbf{C}$ of $C$, given by $\mathbf{f}=0, \mathbf{f} \in \mathbb{C}\{x, y, t\}$. Furthermore we have deformations $\boldsymbol{\ell}(i)$ of the schemes of length $\ell(i)$ for each $i \in T$, given by a subscheme $\boldsymbol{\ell}$. As above we can multiply with the conductor, and this can then be cut out by an element $\mathbf{g} \in \mathbb{C}\{x, y, t\}$. On a general point of the parameter space we require the fiber to have ordinary $m$-tuple points (for possibly several $\mathrm{m}$ ). Recall that $\delta$-constant is equivalent to deforming the parametrization of each branch $C_{i}$. The subscheme $\ell(i)$ has to be deformed in such a way that all inverse images of a singular point on the normalization of $C_{i}$ have to be occupied by a point of the deformed subscheme of $\ell(i)$. Indeed, from the pair $(\mathbf{C}, \ell)$, we can get a space $Y(\mathbf{C}, \ell)$ in $\mathbb{C}^{4}$ by the equation $z \mathbf{f}-\mathbf{g}=0$, whose normalization gives the smoothing $X(\mathbf{C}, \ell)$ of $X(C, \ell)$. The Milnor fiber is obtained by blowing up $\mathbb{C}^{2}$ in the images of the points of the deformed subscheme $\ell(i)$ of $\ell(i)$, and removing the strict transform of $C$. For more precise statement and proofs of the above results, we refer to [JS98], in particular Theorem 4.4, Lemma 4.7 and Proposition 5.1.

Let $\mathbf{C}$ be a one-parameter (with parameter $t$ ) $\delta$-constant deformation of $C$ as above. If $I$ is the conductor of $C$, then the conductor is also deformed in a flat way. Thus we get an ideal $\mathbf{I} \subset \mathbb{C}\{x, y, t\}$. On a general $m$-tuple point of $\mathbf{C}$, this ideal $\mathbf{I}$ is just taking the $(m-1)$ th power of the maximal ideal 


\section{T. DE JONG}

defining this point. The following is an important observation, which follows immediately from the duality theorem for finite maps.

Lemma 3. The ideal $\mathbf{I}$, considered as ideal on the local ring of the smoothing $X(\mathbf{C}, \boldsymbol{\ell})$, is a canonical ideal of $X(\mathbf{C}, \ell)$.

The main purpose of this paper is to prove the following theorem.

Theorem 4. Let $X(\mathbf{C}, \boldsymbol{\ell})$ be a smoothing of a sandwiched singularity as described above. Consider the ideal $\mathbf{I} \subset \mathbb{C}\{x, y, t\}$. Then the canonical algebra of $X(\mathbf{C}, \ell)$ is finitely generated if and only if the symbolic algebra $\bigoplus_{k=0}^{\infty} \mathbf{I}^{(k)}$ is finitely generated.

Remark 5. As the ideal $\mathbf{I}$ is much easier to describe explicitly than the corresponding smoothing of the sandwiched singularity, I think that it is easier to check the finite generatedness of the symbolic algebra. One should beware of the fact that there exist ideals in $\mathbb{C}\{x, y, t\}$ for which the symbolic algebra is not finitely generated!

So, if Kollár's conjecture is false, then Theorem 4 could be of significant help for finding a counterexample. Altogether, it is hoped that Theorem 4 will lead to a better understanding and a possible attack on Kollár's conjecture for sandwiched singularities.

\section{Proof of the theorem}

For lack of a reference, we include a proof of the following proposition which we will need in the proof of Theorem 4.

Proposition 6. Consider an ideal $\mathbf{I} \subset \mathcal{O}_{\mathbb{C}^{3}, 0}$ without embedded primes and let $\Sigma:=V(\mathbf{I})$. The following are equivalent.

1) The symbolic algebra $\bigoplus_{k=0}^{\infty} \mathbf{I}^{(k)}$ is finitely generated.

2) There exists a modification $\pi:(\mathbf{W}, \mathbf{F}) \longrightarrow\left(\mathbb{C}^{3}, \Sigma\right)$ such that:

a) $\mathbf{F}=\pi^{-1}(\Sigma)$ is $\mathbb{Q}$-Cartier and $\pi$-ample;

b) $\pi$ is 'small', that is, $\pi^{-1}(0)$ is one dimensional.

Proof. Suppose $\bigoplus_{k=0}^{\infty} \mathbf{I}^{(k)}$ is finitely generated. Then we can consider the symbolic blowing-up $\mathbf{W}:=$ $\operatorname{Proj}\left(\bigoplus_{k=1}^{\infty} \mathbf{I}^{(k)}\right)$. We have a natural projective morphism $\pi:(\mathbf{W}, \mathbf{F}) \longrightarrow\left(\mathbb{C}^{3}, \Sigma\right)$. As $\bigoplus_{k=1}^{\infty} \mathbf{I}^{(k)}$ is finitely generated, there exists an $m \in \mathbb{N}$ such that $\mathbf{I}^{(k m)}=\left(\mathbf{I}^{(m)}\right)^{k}$. We have $\operatorname{Proj}\left(\bigoplus_{k=0}^{\infty} \mathbf{I}^{(k)}\right)=$ $\operatorname{Proj}\left(\bigoplus_{k=1}^{\infty} \mathbf{I}^{(k m)}\right)$. Thus $\mathbf{W}$ is the blowing-up in the ideal $\mathbf{I}^{(m)}$. So on $\mathbf{W}$ we have the very ample sheaf $\mathcal{O}_{\mathbf{W}}(1)$. Hence $m \mathbf{F}$ is Cartier. Suppose $\pi$ is not small. Then we have an exceptional divisor $E$, and thus a proper inclusion of sheaves $\mathcal{O}_{\mathbf{W}} \varsubsetneqq \mathcal{O}_{\mathbf{W}}(E)$, and thus, for $k \gg 0$, an injection

$$
\varphi: \mathbf{I}^{(k m)}=\pi_{*} \mathcal{O}_{\mathbf{W}}(k) \varsubsetneqq \pi_{*} \mathcal{O}_{\mathbf{W}}(k)(E) .
$$

This is a contradiction as $\mathbf{I}^{(k m)}$ is a reflexive sheaf, and $\varphi$ is an isomorphism outside the codimension two set $(\Sigma, 0) \subset\left(\mathbb{C}^{3}, 0\right)$.

The converse follows, as for some $m$ the ideal $\mathbf{I}^{(m)}$ on $\mathbf{W}$ is invertible, thus $\bigoplus_{k=0}^{\infty} \mathbf{I}^{(k m)}$ is generated by $\mathbf{I}^{(m)}$. Thus $\bigoplus_{k=0}^{\infty} \mathbf{I}^{(k)}$ is finitely generated.

Remark 7. The proposition implies that if, in a specific example, the symbolic algebra is finitely generated, this can be checked in a finite number of steps. Namely, one computes the blowing-up in the ideal $\mathbf{I}^{(k)}$ for higher and higher $k$, until one reaches a small modification of a small neighborhood of 0 in $\mathbb{C}^{3}$. Note that Theorem 4 can be used to prove the finite generatedness of the symbolic algebra of certain non-reduced curves in three-space. 


\section{CANONICAL ALGEBRA OF SMOOTHINGS OF SANDWICHED SINGULARITIES}

Note that if the symbolic algebra is generated by $\mathbf{I}, \ldots, \mathbf{I}^{(m)}$ with $m$ minimal, then $m \mathbf{F}$ is Cartier. Moreover, if $\operatorname{Proj}\left(\bigoplus_{k=1}^{\infty} \mathbf{I}^{(k)}\right)$ is a small modification, then $\mathbf{I}^{(k m)}=\left(\mathbf{I}^{(m)}\right)^{k}$ for all $k$. This can be used to give a proof of the following well-known fact.

Corollary 8. Suppose that $\mathbf{I}=(f, g)$, where $f, g$ is a regular sequence. Then $\mathbf{I}^{(k)}=\mathbf{I}^{k}$ for all $k \in \mathbb{N}$.

Proof. The blowing-up in the ideal $\mathbf{I}$ is given as a subspace of $\mathbb{P}^{1} \times\left(\mathbb{C}^{3}, 0\right)$ whose ideal is given by the homogeneous (in $A, B$ ) kernel of the map

$$
\mathbb{C}[A, B]\{x, y, z\} \longrightarrow \mathbb{C}\{x, y, z\}
$$

which sends $A$ to $f$ and $B$ to $g$. This certainly is a small modification, given by the equation $A g-B f=0$.

Example 9. We consider three-space with coordinates $x, y$ and $z$, and the ideal $\mathbf{I}=(x y, x z, y z)$ describing the coordinate axes. It is easy to show that the symbolic algebra is finitely generated. We in fact blow up in the ideal $\mathbf{I}^{(2)}=\left(x^{2} y^{2}, x^{2} z^{2}, y^{2} z^{2}, x y z\right)$. The space $\mathbf{W}=\operatorname{Proj}\left(\bigoplus_{k=0}^{\infty} \mathbf{I}^{(2)^{k}}\right)$ lies in $\mathbb{P}^{3} \times\left(\mathbb{C}^{3}, 0\right)$ and the ideal is given by the homogeneous kernel (homogeneous in $\left.A, B, C, F\right)$ of the map

$$
\mathbb{C}[A, B, C, F]\{x, y, z\} \longrightarrow \mathbb{C}\{x, y, z\}
$$

which sends $A$ to $x^{2} y^{2}, B$ to $x^{2} z^{2}, C$ to $y^{2} z^{2}$, and $F$ to $x y z$. One computes this kernel to be generated by the following six elements

$$
A B-x^{2} F^{2}, A C-y^{2} F^{2}, B C-z^{2} F^{2}, x y F-z A, x z F-y B, y z F-x C .
$$

For $F=1$, we get that these are given by the $2 \times 2$ minors of the matrix

$$
\left(\begin{array}{lll}
A & x & y \\
x & B & z \\
y & z & C
\end{array}\right)
$$

which generate the ideal of the cone over the Veronese. The inverse image $\pi^{-1}(0)$ is given by plugging in $x=y=z=0$, so given by the equations $A B=A C=B C=0$. These give three projective lines intersecting in one point in three-dimensional projective space. Thus we see that the modification is small.

We now prove one implication of Theorem 4.

Lemma 10. Let $(X(\mathbf{C}, \ell), x)$ be a smoothing of a sandwiched singularity as described above. Consider the conductor ideal $\mathbf{I} \subset \mathbb{C}\{x, y, t\}$ of $\mathbf{C}$. Suppose the canonical algebra of $X(\mathbf{C}, \boldsymbol{\ell})$ is finitely generated. Then the symbolic algebra $\bigoplus_{k=0}^{\infty} \mathbf{I}^{(k)}$ is finitely generated.

Proof. Consider the space $X(\mathbf{C}, \ell)$. The canonical algebra is finitely generated, so we get a small modification of $\mathbf{W}$ of $X(\mathbf{C}, \ell)$, with the property that $K_{\mathbf{W}}$ is $\mathbb{Q}$-Cartier, that is, $\mathbf{I}^{(m)}$ is invertible for some $m$. By the results of [JS98], as mentioned above, every fiber of $X(\mathbf{C}, \ell)$ is obtained by blowing up ideal sheaves. Thus the space $\mathbf{W}$ is a small modification of $\mathbb{C}^{3}$, with $\mathbf{I}^{(m)}$ invertible. Hence by Proposition 6 the symbolic algebra $\bigoplus_{k=0}^{\infty} \mathbf{I}^{(k)}$ is finitely generated.

Now we look, until Remark 17, at the converse statement. This is more difficult. So consider an ideal $\mathbf{I} \subset \mathbb{C}\{x, y, t\}$, and suppose that $\bigoplus_{k=0}^{\infty} \mathbf{I}^{(k)}$ is finitely generated. Thus we have a modification $\pi:(\mathbf{W}, \mathbf{F}) \longrightarrow\left(\mathbb{C}^{3}, \Sigma\right)$ by the proposition.

We now look at the restriction $W$ of $\mathbf{W}$ to the fiber $t=0$. So we get a modification $\pi:(W, F) \longrightarrow$ $\left(\mathbb{C}^{2}, 0\right)$. The space $W$ is equal to $\operatorname{Proj}\left(\bigoplus_{k=0}^{\infty} \mathbf{I}_{t=0}^{(k)}\right)$. We write $I(k):=\mathbf{I}_{t=0}^{(k)}$. Note that $I(k)$ not only depends on $I$, but also on the particular deformation of the curve $C$ we are looking at. We first look at two examples, in order to see what to expect. 


\section{T. DE JONG}

Example 11. In Example 9 we put $z=x+y-t$, and view the curve consisting of three lines in $\mathbb{C}^{3}$, given by the ideal $(x y, x(x+y-t), y(x+y-t))$ as a deformation of the ideal $(x y, x(x+y), y(x+y))=$ $\left(x^{2}, x y, y^{2}\right)$. We take $f=x y(x+y)$, and $\mathbf{C}$ given by $x y(x+y-t)=0$. Thus $\mathbf{C}$ is the cone over three lines in $\mathbb{P}^{2}$. In this case, we have that $W$ is given by the ideal given in (1) by plugging in $(x+y)$ instead of $z$. The result is:

$$
\begin{gathered}
A B-x^{2} F^{2}, A C-y^{2} F^{2}, B C-(x+y)^{2} F^{2}, x y F-(x+y) A, \\
x(x+y) F-y B, y(x+y) F-x C .
\end{gathered}
$$

For $F=1$ we get a plane section of the cone over the Veronese, hence a cone over the rational normal curve of degree four. (Of course one can calculate this directly.) The exceptional divisor we already saw to be the union of the three $\mathbb{P}^{1}$ intersecting in the singular point of the cone.

We plug in $F=0$ and get the ideal

$$
A B, A C, B C,(x+y) A, y B, x C .
$$

This is the ideal of a space in $\mathbb{P}^{2} \times\left(\mathbb{C}^{2}, 0\right)$. We have three possibilities:

1) $(A: B: C)=(1: 0: 0) ; \quad(x+y)=0 ;$

2) $(A: B: C)=(0: 1: 0) ; \quad y=0$;

3) $(A: B: C)=(0: 0: 1) ; \quad x=0$.

Thus we see that the three lines given by $x y(x+y)=0$ separate on the modification $W$, and intersect in non-singular points of the modification.

Example 12. Consider the curve $C$ given by $f(x, y)=y^{3}-x^{4}=0$. The conductor is given by $I=$ $(x, y)^{2}$. From the theory of plane curve singularities we know that $C$ has a $\delta$-constant deformation to a curve with three ordinary double points. The total space $\mathbf{C}$ with equation $\mathbf{f}=0$ is smooth and the conductor $\mathbf{I}$ is reduced. It follows that $\mathbf{I}^{(2)}=\mathbf{I}^{2}+(\mathbf{f})$, and $\mathbf{I}^{(2 k)}=\left(\mathbf{I}^{(2)}\right)^{k}$. Restricting to the zero fiber, we have to blow up the ideal $(x, y)^{4}+(f)$, or what is the same, in the ideal $\left(x^{4}, x^{3} y, x^{2} y^{2}, y^{3}\right)$. This turns out to be a complete ideal, and the blowing-up is given by the homogeneous kernel (homogeneous in $A, B, C, D$ ) of the map

$$
\mathbb{C}[A, B, C, D]\{x, y\} \longrightarrow \mathbb{C}\{x, y\}
$$

which sends $A$ to $x^{4}$ etc. This kernel is given by the six elements

$$
A C-B^{2}, x A D-B C, x B D-C^{2}, y A-x B, y B-x C, y C-x^{2} D .
$$

One can compute that for $D=1$ we again have a cone over the rational normal curve of degree four. The exceptional set is given by

$$
A C-B^{2}, B C, C^{2}
$$

The reduced exceptional set is thus a $\mathbb{P}^{1}$ with homogeneous coordinates $(A: D)$. At infinity, that is, the point with homogeneous coordinates $(1: 0)$, one computes that one has an $A_{2}$-singularity. So in total we have an exceptional $\mathbb{P}^{1}$, with two singularities on it.

We compute the strict transform of $C$, given by $y^{3}-x^{4}$. This will turn out to go through the point $(A: D)=(1: 1)$ on the exceptional divisor. Indeed, plugging in $A=D=1$ in our generators of the ideal, we are left with (minimally) three elements

$$
C-B^{2}, x-B C, y-x B .
$$

Upon eliminating $C$ and $B$ we indeed get the element $y^{3}-x^{4}$. Note that the strict transform of $C$ is the normalization of $C$, which does not go through any of the singular points of the modification.

Proposition 13. The strict transform $\bar{C}$ of $C$ under $\pi:(W, F) \longrightarrow\left(\mathbb{C}^{2}, 0\right)$ is smooth. 


\section{CANONICAL ALGEBRA OF SMOOTHINGS OF SANDWICHED SINGULARITIES}

Proof. The conductor $I$ is on the normalization of $C$ a principal ideal, and thus we have that $I^{k}=I(k)$ modulo $f$. Thus the strict transform is just the blowing-up of the conductor, hence equal to the normalization.

Proposition 14. Consider an ideal $J \subset \mathbb{C}\{x, y\}$ of finite codimension. Let $Z$ be the blowing-up of $J$. Let $f \in \mathbb{C}\{x, y\}$. Suppose $f=0$ has strict transform on $Z$ that is smooth. Let $h \in \mathbb{C}\{x, y\}$, and suppose the strict transforms of $h=0$ and $f=0$ on $Z$ do not intersect. Consider $J^{\prime}:=J+(h f)$, and $Z^{\prime}$ be the blowing-up of $J^{\prime}$. Let $\bar{C}$ be the strict transform of $f=0$ on $Z^{\prime}$, and $F$ be the exceptional divisor. Then $\bar{C}$ is smooth, and $\bar{C} \cap F$ consists of smooth points of $Z^{\prime}$.

Proof. We write $J=\left(g_{1}, \ldots, g_{\alpha}\right)$. The space $Z$ is a subset of $\left(\mathbb{C}^{2}, 0\right) \times \mathbb{P}^{\alpha-1}$. We take homogeneous coordinates $G_{1}, \ldots, G_{\alpha}$ of $\mathbb{P}^{\alpha-1}$. Then $Z$ is given by a set of equations which are homogeneous in the $G_{i}$, and is given by the kernel of the map

$$
\mathbb{C}\left[G_{1}, \ldots, G_{\alpha}\right]\{x, y\} \longrightarrow \mathbb{C}\{x, y\},
$$

that sends $G_{i}$ to $g_{i}$. The strict transform $\bar{C}$ of $f=0$ is given similarly by the kernel of the map

$$
\alpha: \mathbb{C}\left[G_{1}, \ldots, G_{\alpha}\right]\{x, y\} \longrightarrow \mathbb{C}\{x, y\} /(f),
$$

which sends $G_{i}$ to $g_{i}$.

The space $Z^{\prime}$ is given as a subset of $\left(\mathbb{C}^{2}, 0\right) \times \mathbb{P}^{\alpha}$, where we have homogeneous coordinates $G_{1}, \ldots, G_{\alpha}, H$ on $\mathbb{P}^{\alpha}$, as the kernel of the map

$$
\beta: \mathbb{C}\left[G_{1}, \ldots, G_{\alpha}, H\right]\{x, y\} \longrightarrow \mathbb{C}\{x, y\},
$$

which sends $G_{i}$ to $g_{i}$ and $H$ to $h f$. Similarly, the strict transform of $C$ on $Z^{\prime}$ is given by the kernel of the map induced by $\beta$ to $\mathbb{C}\{x, y\} /(f)$. As $\beta(H)=h f$, it follows that this strict transform is smooth. Furthermore, on $Z^{\prime}$ the space $H=0$ gives the total transform of $h f=0$ on $Z^{\prime}$. Because the strict transforms of $h=0$ and $f=0$ do not intersect on $Z$, they do not intersect on $Z^{\prime}$ either. It follows that locally at points of $F \cap \bar{C}$ on $Z^{\prime}$ the smooth space $\bar{C}$ is given by a single equation. This can only happen at smooth points of $Z^{\prime}$.

Proposition 15. Consider the blowing-up of the ideal $I(m)$ of $\mathbb{C}^{2}$. The strict transform $\bar{C}$ intersects $F$ only in smooth points of $W$.

Proof. We already noted that $I(m)=I^{m} \bmod f$. The blowing-up of $I^{m}$ is isomorphic to the blowingup of $I$, and the strict transform of $C$ is smooth on the blowing-up. To come from $I^{m}$ to $I(m)$ we only need finitely many more generators which are all divisible by $f$. We apply Proposition 14 inductively, and thus we have to show that those extra generators $h_{1} f, \ldots, h_{s} f$ can be taken such that the strict transform of $h_{i}=0$ does not intersect the strict transform of $f=0$ on the appropriate blow up. This we can achieve by replacing (if necessary) $h_{i} f$ by $\left(h_{i}+L^{d}\right) f$ for $d \gg 0$, and $L=a x+b y$ a general linear form. As $d \gg 0$ we have $L^{d} f \in I^{m}$, and as $L$ is general, the strict transform of $L=0$ does not intersect the strict transform of $f=0$.

We now give the construction of the modification of a smoothing of a sandwiched singularity, in order to prove the other implication of Theorem 4.

Proposition 16. Let $X(\mathbf{C}, \boldsymbol{\ell})$ be a smoothing of the sandwiched singularity $X(C, \ell)$, and let $\mathbf{I}$ be the conductor. Suppose that the symbolic algebra of $\mathbf{I}$ is finitely generated, and consider the modification $(\mathbf{W}, \mathbf{F}) \longrightarrow\left(\mathbb{C}^{3}, \Sigma\right)$. It follows from Proposition 15 that the strict transform of $\mathbf{C}$ on $\mathbf{W}$ is smooth, so we can see $\boldsymbol{\ell}$ as a subscheme of $\mathbf{W}$. Let $D$ be those irreducible components of $\boldsymbol{\ell}$ whose image in $\mathbf{C}$ is not contained in $V(\mathbf{I})$. Let $\mathbf{W}_{D}$ be the blowing-up of $\mathbf{W}$ in $D$. Then there is a $\operatorname{map} \pi: \mathbf{W}_{D} \longrightarrow X(\mathbf{C}, \boldsymbol{\ell})$ satisfying the conditions of Proposition 2. In particular the 'if' statement in Theorem 4 holds. 


\section{CANONICAL ALGEBRA OF SMOOTHINGS OF SANDWICHED SINGULARITIES}

Proof. We give a different description of the space $\mathbf{W}_{D}$. Restricting the curve $D$ to the special fiber, we get a finite union of fat points. Thus the special fiber $W^{\prime}$ of $\mathbf{W}_{D}$ is the blowing-up of $W$ of a finite number of fat points. We note that $W^{\prime}$ is a modification of a sandwiched space $X\left(C, \ell^{\prime}\right)$ for some $\ell^{\prime}$. (We will see in a moment that $\ell^{\prime}=\ell$.) Indeed, resolve all the singularities of $W^{\prime}$, which gives an embedded resolution $\bar{W}$ of $C$. Then we blow down on $\bar{W}$ all compact curves not intersecting any branch of the strict transform $\bar{C}$ of $C$ on $\bar{W}$. This gives a sandwiched space $X\left(C, \ell^{\prime}\right)$, and a map $p: \bar{W} \longrightarrow X\left(C, \ell^{\prime}\right)$. The map $p$ factorizes over $W^{\prime}$, as all singularities of $W^{\prime}$ lie on exceptional curves of the map $W \longrightarrow \mathbb{C}^{2}$. Hence we get a map $p^{\prime}: W^{\prime} \longrightarrow X\left(C, \ell^{\prime}\right)$. In particular we see that $p^{\prime}$ is a point modification Note that, by construction, the space $W^{\prime}$ only has $q G$-singularities or

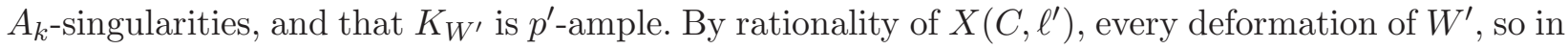
particular $\mathbf{W}_{D}$, blows down to a deformation of $X\left(C, \ell^{\prime}\right)$. From the explicit description of the Milnor fiber [JS98, ch. 5], recalled at the beginning of this paper, it follows that $\ell=\ell^{\prime}$, and also we get the map $\pi: \mathbf{W}_{D} \longrightarrow X(\mathbf{C}, \ell)$. This explicit description of the Milnor fiber also shows that $\pi$ is small, as it is an isomorphism on the general fiber. The two properties b and $c$ of part 2 of Proposition 2 follow from the corresponding properties of the space $(\mathbf{W}, \mathbf{F}) \longrightarrow\left(\mathbb{C}^{3}, \Sigma\right)$. This concludes the proof.

Remark 17. I have tried for a long time to prove Theorem 4 directly, by comparing the ideal $\mathbf{I}^{(k)}$ with the $k$ th symbolic power of the canonical ideal on the smoothing of the sandwiched singularity. Unfortunately, I did not succeed, so I decided to prove Theorem 4 as done in this paper.

\section{REFERENCES}

Art70 M. Artin, Algebraic construction of Brieskorn resolutions, J. Algebra 29 (1970), 88-135.

Hir83 H. Hironaka, On Nash blowing up, in Arithmetic and geometry II (Birkhäuser, Basel, 1983), 103-111.

JS91 T. de Jong and D. van Straten, On the base space of a semi-universal deformation of rational quadruple points, Ann. of Math. (2) 134 (1991), 653-678.

JS98 T. de Jong and D. van Straten, Deformation theory of sandwiched singularities, Duke Math. J. 95 (1998), 451-522.

Kaw88 Y. Kawamata, Crepant blowing-up of 3-dimensional canonical singularities and its application to degenerations of surfaces, Ann. of Math. (2) 127 (1988), 93-163.

Kol91 J. Kollár, Flips, flops, minimal models, etc., in Surveys in differential geometry, Lehigh University, Bethlehem, PA (International Press, Boston, MA, 1991), 195-279.

KS88 J. Kollár and N. Shepherd-Barron, Threefolds and deformations of surface singularities, Invent. Math. 91 (1988), 299-338.

Lip69 J. Lipman, Rational singularities with applications to algebraic surfaces and unique factorization, Publ. Math. Inst. Hautes Études Sci. 36 (1969), 195-279.

Spi90 M. Spivakovsky, Sandwiched singularities and desingularization of surfaces by normalized Nash transformations, Ann. of Math. (2) 131 (1990), 411-491.

Ste91 J. Stevens, Partial resolutions of rational quadruple points, Int. J. Math. 2 (1991), 205-221.

T. de Jong dejong@mathematik.uni-mainz.de

FB 17 Mathematik und Informatik, Johannes Gutenberg-Universität Mainz, Staudingerweg 9, D-55128 Mainz, Germany 